\title{
Supramolecular Additive-Initiated Controlled Atom Transfer Radical Polymerization of Zwitterionic Polymers on Ureido-pyrimidinone- Based Biomaterial Surfaces
}

\author{
Bastiaan D. Ippel, ${ }^{\#}$ Muhabbat I. Komil, ${ }^{\#}$ Paul A. A. Bartels, Serge H. M. Söntjens, Roy J. E. A. Boonen, \\ Maarten M. J. Smulders, and Patricia Y. W. Dankers*
}

Cite This: Macromolecules 2020, 53, 4454-4464

Read Online

ACCESS | Lلll Metrics \& More | 回 Article Recommendations | st Supporting Information

ABSTRACT: Surface-initiated controlled radical polymerization is a popular technique for the modification of biomaterials with, for example, antifouling polymers. Here, we report on the functionalization of a supramolecular biomaterial with zwitterionic poly(sulfobetaine methacrylate) via atom transfer radical polymerization from a macroinitiator additive, which is embedded in the hard phase of the ureido-pyrimidinone-based material. Poly(sulfobetaine methacrylate) was successfully polymerized from these surfaces, and the polymerized sulfobetaine content, with corresponding antifouling properties, depended on both the macroinitiator additive concentration and polymerization time. Furthermore, the polymerization from the macroinitiator additive was successfully translated to functional electrospun scaffolds, showing the potential for this functionalization strategy in supramolecular material systems.

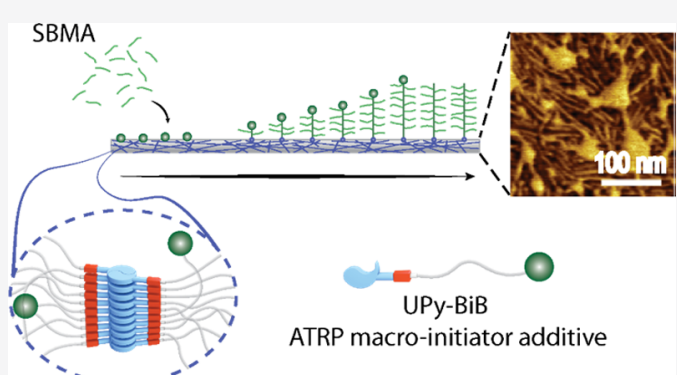

\section{INTRODUCTION}

Antifouling functionalization strategies are of interest for a variety of applications, ranging from the prevention of biofouling in marine environments to the restriction of fouling of blood components in vascular grafts. ${ }^{1,2}$ Hydrophilic surface functionalization is a well-established method to decrease undesired fouling for biomaterials ${ }^{3,4}$ and is hypothesized to be effective through the formation of a thin but stable hydrated layer that serves as a barrier between the material and the environment. ${ }^{5}$ In recent years, surface functionalization with zwitterionic polymers has gained more traction for the purpose of generating antifouling properties. ${ }^{6}$ Their ability to repel, for instance, protein adsorption, exceeds that of classic hydrophilic polymers such as poly(ethylene glycol) (PEG) through a stronger interaction between zwitterions and water molecules. ${ }^{7,8}$ Monomers with intramolecular opposing charges on which such polymers are based include carboxybetaine, sulfobetaine, and phosphorylcholine derivatives. ${ }^{6}$ The antifouling properties of zwitterionic layers depend on the design of the zwitterionic moiety in terms of distances between the cations and anions, the overall polymer morphology and molecular weight, and functionalization density. ${ }^{1,9}$

Several controlled polymerization strategies can be applied to functionalize biomaterial surfaces with zwitterionic polymers, among which radical polymerization techniques such as surface-initiated atom transfer radical polymerization (SIATRP) can yield highly antifouling layers. ${ }^{3,10,11}$ Furthermore, surface-initiated controlled radical polymerization (SI-CRP) allows for precise control of molecular weight and polymer morphology, as well as grafting density. ${ }^{12,13}$

Methods for SI-CRP are commonly developed on model substrates such as silica or flat and thin polymer layers. ${ }^{14}$ The availability of a highly reactive initiating group on the surface of these materials is a prerequisite. Initiators can be simply covalently conjugated to a surface ${ }^{15-17}$ or attached through catechol-mediated chemistry. ${ }^{18,19}$ Both these methods can be applied to functional constructs such as filtration membranes and biological scaffolds, ${ }^{20-25}$ but they require additional processing steps after fabrication prior to polymerization.

Alternatively, zwitterionic amphiphilic additives, where a macromolecular hydrophobic block facilitates anchoring in the base material through hydrophobic interactions, have been previously used to directly incorporate zwitterionic functionality. $^{26-31}$ However, control over the morphology of the zwitterionic layer is limited using this method.

Inclusion of additives with the initiating groups before fabrication, in a similar manner to the formulation of everyday polymeric materials, can overcome the need for extra postfabrication modification of the material. One approach

Received: January 21, 2020

Revised: April 21, 2020

Published: May 26, 2020 
A

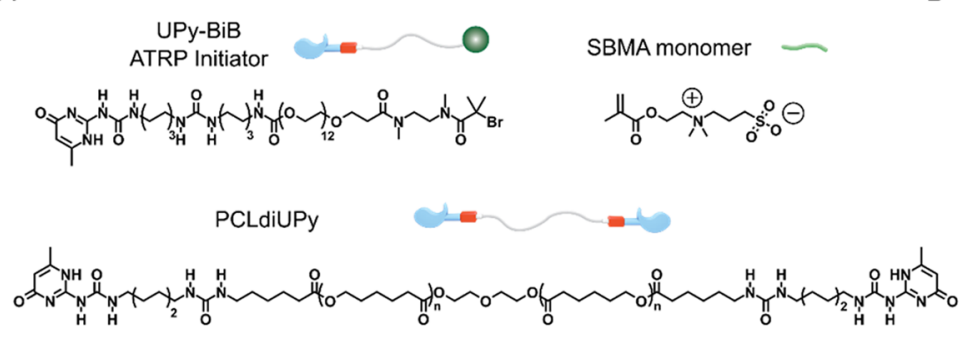

B

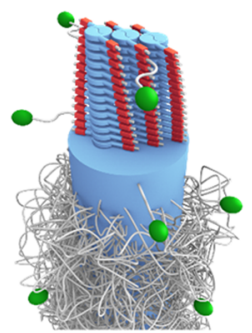

C
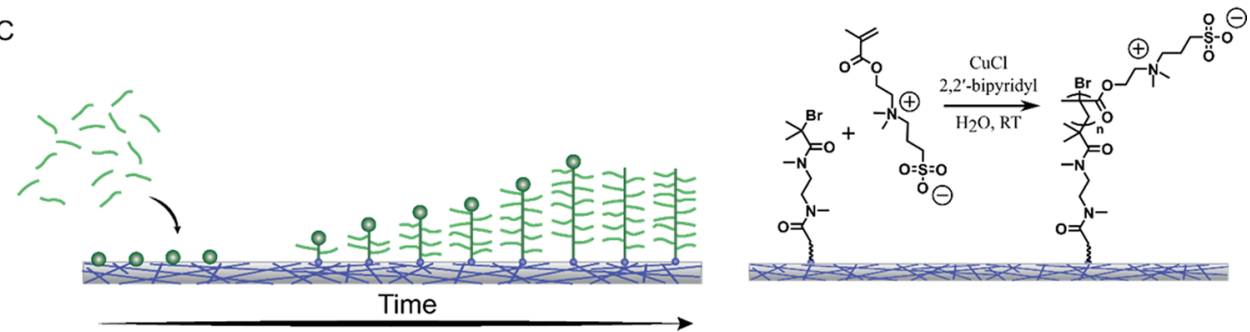

Figure 1. Schematic overview of the approach used in this study. (A) Structural and graphical representations of the UPy-modified polycaprolactone (PCLdiUPy) supramolecular base material, the UPy-BiB macroinitiator additive, and the sulfobetaine methacrylate (SBMA) monomer. (B) Schematic representation of the fibrous hard phase formed through UPy dimerization and assembly. (C) Schematic representation of SI-ATRP with SBMA from the UPy-BiB initiator additive.

uses a mixture of nonfunctionalized macromolecular building blocks and macromolecules functionalized with initiating groups, for the fabrication of, e.g., porous membranes. ${ }^{32-34}$ From the available initiators at the surface of the membranes or electrospun scaffolds, antifouling polymers were polymerized, thereby retaining the control over the brush properties. ${ }^{35}$ Similarly, in a peptide-amphiphile (PA)-based system, PAs with a tertiary bromide $(\mathrm{BiB})$ were blended with regular PAs, which allowed for ATRP from the self-assembled nanofibers. ${ }^{36}$ For biomaterial development, we propose that the interactions between the structural material and the functionalized component, or additive, should be managed with care. Additional interactions, such as hydrogen bonding, introduce a higher degree of specificity.

A well-known example of a material system that relies on hydrogen bonding features the self-complementary 2-ureido$4[1 H]$-pyrimidinone (UPy) moiety. ${ }^{37-39}$ UPy-UPy dimers can self-assemble into nanofibrous structures through $\pi-\pi$ interactions, which are further stabilized by additional hydrogen bonds between urea groups (Figure 1B). ${ }^{40,41}$ In solid thin films, these assemblies can be visualized well with, e.g., atomic force microscopy (AFM) (Figure 2). ${ }^{40,41}$

Previously, these supramolecular materials were specifically functionalized with additives that contain matching supramolecular hydrogen-bonding motifs, for antifouling properties, ${ }^{42-45}$ antimicrobial activity, ${ }^{46}$ additional bioactivity, ${ }^{44,47-50}$ and postfunctionalization. ${ }^{51,52}$ The addition of the matching hydrogen-bonding motif prevents excessive erosion and phase separation of peptide-functionalized additive from the bulk, while the activity of the peptides is retained. ${ }^{46,53}$

Here, we employ the hydrogen-bonding UPy moiety to specifically incorporate a UPy macroinitiator for SI-ATRP into the self-assembled UPy fibers, to increase the functionalization potential for this class of materials.

A newly synthesized UPy-ATRP macroinitiator additive that contains a tertiary bromide (UPy-BiB) was mixed with UPymodified polycaprolactone (PCLdiUPy) (Figure 1). Thin films were cast from these mixtures for initial screening of the reactivity of the macromolecular initiator at the surface of the

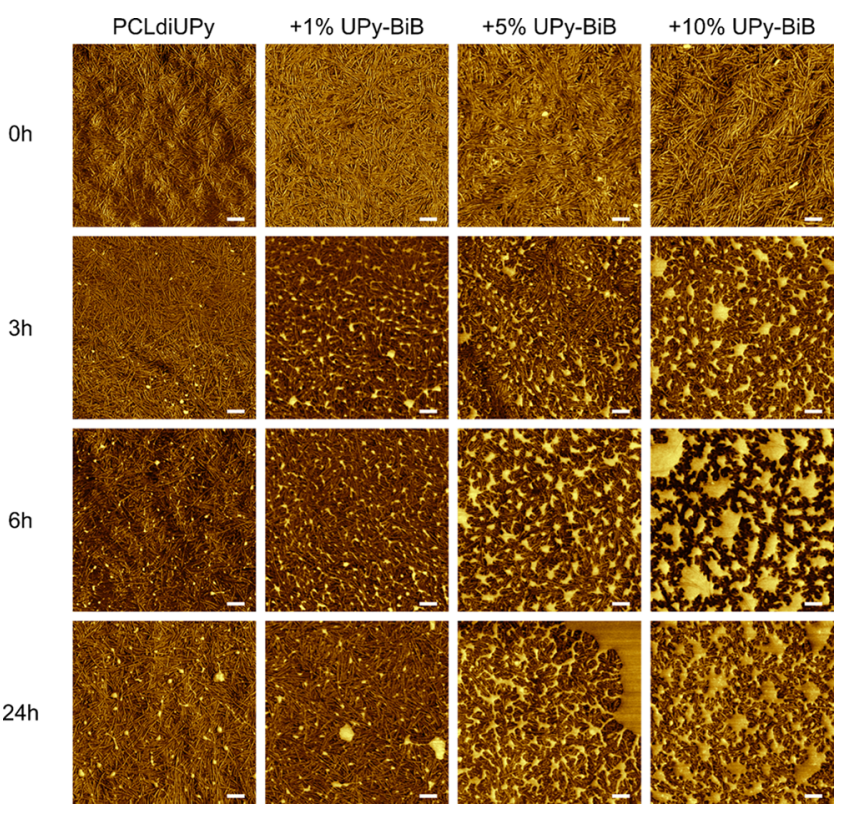

Figure 2. AFM phase micrographs of solution-cast films of PCLdiUPy with $0,1,5$, and $10 \% \mathrm{UPy}-\mathrm{BiB}$ macroinitiator additive before and after 3,6 , and $24 \mathrm{~h}$ of polymerization on the surfaces. The scale bars indicate $100 \mathrm{~nm}$.

supramolecular material. Sulfobetaine methacrylate (SBMA) monomers were polymerized by means of SI-ATRP from the additive that was embedded in the hard phase of the constructs (Figure 1). The surfaces were characterized with water contact angle (WCA) measurements, X-ray photoelectron spectroscopy (XPS), and atomic force microscopy (AFM), followed by functional cell adhesion assays. Furthermore, functional porous scaffolds were fabricated from the material mixtures with the $\mathrm{UPy}-\mathrm{BiB}$ additive via electrospinning, to demonstrate the applicability of the approach described here. 


\section{EXPERIMENTAL SECTION}

Materials. Unless stated otherwise, all reagents were used without further purification. tert-Butyl phenyl carbonate (98\%), $N, N^{\prime}$ dimethyl-1,2-diaminoethane (99\%), N,N'-diisopropylethylamine (DIPEA, 99.5\%), $\alpha$-bromoisobutyryl bromide (98\%), $N$-methylmorpholine (NMM, $\geq 99.5 \%)$, copper(I) chloride ( $\mathrm{CuCl}, \geq 99.99 \%)$, copper(II) chloride $\left(\mathrm{CuCl}_{2}, 97 \%\right)$, and 2,2'-bipyridyl (bpy, $\geq 99 \%$ ) were obtained from Sigma-Aldrich. Hydrogen chloride $(\mathrm{HCl})$ solution in dioxane was purchased from Acros Organics. 2-(N-3Sulfopropyl- $N, N$-dimethyl ammonium)ethyl methacrylate (SBMA, $\geq 98 \%)$ was obtained from Merck. $N, N, N^{\prime}, N^{\prime}$-Tetramethyl-O-( $1 H$ benzotriazol-1-yl)uronium hexafluorophosphate (HATU) was purchased from Novabiochem. UPy-OEG12-COOH was synthesized as described previously, ${ }^{54}$ and UPy-modified polycaprolactone (PCLdiUPy) was purchased from SyMo-Chem. Copper(I) chloride $(\geq 98 \%)$ was purified by vigorous stirring in acetic acid three times, washed with ethanol multiple times, and dried under vacuum overnight. $^{55}$

Chloroform (>99.8\%), diethyl ether (DEE, >99.5\%), dichloromethane (DCM, >99.9\%), N,N-dimethylformamide (DMF, >99.8\%), dioxane (>99.8\%), ethanol (EtOH, $>99.9 \%)$, ethyl acetate $(>99.8 \%)$, heptane (>95\%), and methanol $(>99.8 \%)$ were purchased from Biosolve. 1,1,1,3,3,3-Hexafluoroisopropanol (HFIP, >99\%) was obtained from Sigma-Aldrich. Hydrochloric acid $(\mathrm{HCl})$, sodium hydroxide $(\mathrm{NaOH})$, and sodium carbonate $\left(\mathrm{Na}_{2} \mathrm{SO}_{4}\right)$ were purchased from VWR, Acros Organics, and Fischer Scientific, respectively. Dry DCM was obtained by drying over a $3 \AA$ molecular sieve overnight.

Instrumentation. NMR measurements for characterization of the compounds were performed using either a Bruker UltraShield 400 $\mathrm{MHz}$ or a Varian Mercury $400 \mathrm{MHz}$ spectrometer using $400 \mathrm{MHz}$ for ${ }^{1} \mathrm{H}$ and $100 \mathrm{MHz}$ for ${ }^{13} \mathrm{C}$ measurements. Proton and carbon chemical shifts were documented in parts per million ( $\mathrm{ppm}$ ) downfield from trimethylsilane (TMS), using the resonance frequency of the deuterated solvent as the internal standard. Abbreviations reported for the multiplets are s: singlet; t: triplet; and $\mathrm{m}$ : multiplet. Liquid chromatography/mass spectrometry (LC/MS) measurements were performed on a system using Shimadzu LC-10AD VP liquid chromatography pumps equipped with an Alltima C18 $3 \mu \mathrm{m}$ (50 $\mathrm{mm} \times 2.1 \mathrm{~mm})$ reversed-phase column, a diode array detector (Thermo Finnigan Surveyor PDA Plus detector), and an Ion-Trap (Thermo Scientific LCQ Fleet). This system used a wateracetonitrile mobile phase enriched with $0.1 \% \mathrm{v} / \mathrm{v}$ formic acid.

Synthesis of tert-Butyl Methyl(2-(methylamino)ethyl)carbamate (1) (Scheme S1). In a $250 \mathrm{~mL}$ round-bottom flask, tert-butyl phenyl carbonate $\left(9.52 \mathrm{~g}, 49 \mathrm{mmol}, 1\right.$ equiv) and $N, N^{\prime}$ dimethyl-1,2-diaminoethane (4.32 g, $49 \mathrm{mmol}, 1$ equiv) were dissolved in EtOH $(150 \mathrm{~mL})$. The mixture was stirred overnight at $95{ }^{\circ} \mathrm{C}$ under an argon atmosphere. The reaction mixture was concentrated under reduced pressure, to afford a faint brown viscous liquid, and redissolved in water $(50 \mathrm{~mL})$. With $1 \mathrm{M}$ aqueous $\mathrm{HCl}$, the solution was adjusted to $\mathrm{pH} 2-3$ and washed with DCM $(4 \times 150$ $\mathrm{mL}$ ). The aqueous phase was adjusted to $\mathrm{pH} 10$ with $1 \mathrm{M} \mathrm{NaOH}$ and washed with DCM $(4 \times 150 \mathrm{~mL})$. The organic phases were combined and dried with $\mathrm{Na}_{2} \mathrm{SO}_{4}$ and concentrated under reduced pressure, resulting in a faint yellow viscous liquid $(5.51 \mathrm{~g}, 29.3 \mathrm{mmol}, 60 \%$ yield).

${ }^{1} \mathrm{H}$ NMR (400 MHz, $\mathrm{CDCl}_{3}$ ) $\delta 3.33$ (br, t, $\left.2 \mathrm{H}\right), 2.88(\mathrm{~s}, 3 \mathrm{H}), 2.74$ (br, t, $2 \mathrm{H}), 2.45(\mathrm{~s}, 3 \mathrm{H}), 1.46(\mathrm{~s}, 9 \mathrm{H})$.

Synthesis of tert-Butyl (2-(2-Bromo-N,2dimethylpropanamido)ethyl)(methyl)carbamate (2) (Scheme S2). In a $100 \mathrm{~mL}$ round-bottom flask, 1 (2.00 g, $10.6 \mathrm{mmol}, 1$ equiv) and DIPEA (5.54 g, $31.8 \mathrm{mmol}, 3$ equiv) were dissolved in dry DCM $(10 \mathrm{~mL})$ under an argon atmosphere at $0{ }^{\circ} \mathrm{C}$. A solution of $\alpha$ bromoisobutyryl bromide ( $3.66 \mathrm{~g}, 15.9 \mathrm{mmol}, 1.5$ equiv) in dry DCM $(10 \mathrm{~mL})$ was added dropwise to the round-bottom flask. The mixture was stirred overnight at room temperature under an argon atmosphere. The reaction mixture was concentrated under reduced pressure, redissolved in chloroform $(20 \mathrm{~mL})$, and concentrated under reduced pressure. The resulting viscous liquid was precipitated with DEE $(100 \mathrm{~mL})$, and the product was isolated by flash column chromatography (1:1 heptane/ethyl acetate), resulting in a brown viscous liquid $(2.27 \mathrm{~g}, 6.73 \mathrm{mmol}, 63 \%$ yield $)$.

${ }^{1} \mathrm{H}$ NMR (400 MHz, $\left.\mathrm{CDCl}_{3}\right) \delta 3.56-3.32(\mathrm{br}, \mathrm{m}, 7 \mathrm{H}), 2.90(\mathrm{br}, \mathrm{s}$, $3 \mathrm{H}), 1.97 \mathrm{ppm}$ (br, m, 6H), 1.45 (br, s, 9H).

${ }^{13} \mathrm{C}$ NMR $\left(101 \mathrm{MHz}, \mathrm{CDCl}_{3}\right) \delta 170.30,155.81,77.36,56.96$, 49.11, 48.28, 46.16, 35.14, 32.47.

LC/MS: calcd for $\mathrm{C}_{13} \mathrm{H}_{25} \mathrm{BrN}_{2} \mathrm{O}_{3}: 336.10 \mathrm{~g} / \mathrm{mol}$, found: $127.08[\mathrm{M}$ $+\mathrm{H}+2 \mathrm{Na}]^{3+}, 206.17\left[-\mathrm{NCH}_{3} \mathrm{COO}-\right.$ tert-butyl], $239.00[-\mathrm{BOC}]$, 279.17 [-tert-butyl], $361.08[\mathrm{M}+\mathrm{Na}]^{+}$.

Synthesis of 2-Bromo- $N, 2$-dimethyl- $N$-(2-(methylamino)ethyl)propanamide (3) (Scheme S3). To a solution of 2 (0.10 $\mathrm{g}, 0.30 \mathrm{mmol}, 1$ equiv) in dioxane $(0.30 \mathrm{~mL})$, a solution of $\mathrm{HCl}$ in dioxane $(0.30 \mathrm{~mL})$ was added and stirred overnight at room temperature under an argon atmosphere. The product was isolated by precipitation in cold DEE $(5 \mathrm{~mL})$ and collected by centrifugation. The mixture was redissolved in dioxane $(0.5 \mathrm{~mL})$, precipitated in cold DEE $(5 \mathrm{~mL})$, and centrifuged, resulting in faint yellow hygroscopic solid (0.69 mg, $0.29 \mathrm{mmol}$, $97 \%$ yield).

${ }^{1} \mathrm{H}$ NMR $\left(400 \mathrm{MHz}, \mathrm{CDCl}_{3}\right) \delta 3.83(\mathrm{br}, \mathrm{s}, 2 \mathrm{H}), 3.42(\mathrm{br}, \mathrm{s}, 3 \mathrm{H})$, $3.24(\mathrm{t}, 2 \mathrm{H}), 2.78(\mathrm{~s}, 3 \mathrm{H}), 2.01(\mathrm{~s}, 6 \mathrm{H})$.

${ }^{13} \mathrm{C}$ NMR $\left(101 \mathrm{MHz}, \mathrm{CDCl}_{3}\right) \delta 67.09,65.86,56.67,47.69,46.98$, 33.32, 32.36, 15.28 .

LC/MS: calcd for $\mathrm{C}_{8} \mathrm{H}_{17} \mathrm{BrN}_{2} \mathrm{O}: 236.05 \mathrm{~g} / \mathrm{mol}$, found: $127.08[\mathrm{M}$ $+\mathrm{H}+2 \mathrm{Na}]^{3+} 206.17\left[-\mathrm{NCH}_{3} \mathrm{COO}-\right.$ tert-butyl $], 239.00[\mathrm{M}+\mathrm{H}]^{+}$. Due to bromine atom isotopes, mass peak splitting was observed. Only the highest intensity values of the isotope pattern were reported here.

Synthesis of UPy-BiB (Scheme S4). HATU (48 mg, $12.6 \mathrm{mmol}$, 1.5 equiv) and $N$-methylmorpholine $(18.5 \mathrm{~mL}, 1.68 \mathrm{mmol}, 20$ equiv) were added to a solution of UPy-OEG12-COOH $(65 \mathrm{mg}, 0.08$ mmol, 1 equiv) in DMF ( $4 \mathrm{~mL}$ ) and allowed to stir for $5 \mathrm{~min}$ at room temperature under an argon atmosphere. Subsequently, a solution of $3(24 \mathrm{mg}, 0.10 \mathrm{mmol}, 1.2$ equiv) in DMF $(0.50 \mathrm{~mL})$ was added to the preactivated carboxylic acid mixture and stirred overnight. The product was isolated by precipitation in cold DEE $(50 \mathrm{~mL})$ and collected by centrifugation. The mixture was redissolved in methanol $(5 \mathrm{~mL})$, precipitated in cold DEE $(50 \mathrm{~mL})$, and centrifuged $(3500$ $\mathrm{rpm}, 10 \mathrm{~min})$, resulting in a faint yellow solid $(59 \mathrm{mg}, 0.05 \mathrm{mmol}$, $55 \%$ yield $)$.

${ }^{1} \mathrm{H}$ NMR $\left(400 \mathrm{MHz}, \mathrm{D}_{2} \mathrm{O}\right) \delta 5.98,4.19,3.70,3.36,3.25,3.10,2.97$, $2.74,2.68,2.26,1.96,1.94,1.92,1.56,1.48,1.36,1.31,1.19$.

LC/MS: calcd for $\mathrm{C}_{55} \mathrm{H}_{102} \mathrm{BrN}_{9} \mathrm{O}_{19}: 1271.65 \mathrm{~g} / \mathrm{mol}$, found: 637.92 $[\mathrm{M}+2 \mathrm{H}]^{+}, 1274.58[\mathrm{M}+\mathrm{H}]^{+}$. Due to bromine atom isotopes, mass peak splitting was observed. Only the highest intensity values of the isotope pattern were reported here.

Preparation of Polymer Films. PCLdiUPy (molecular weight, $2650 \mathrm{~g} / \mathrm{mol}$ ) and the additive UPy-BiB were dissolved separately in HFIP at concentrations of $7.55 \mathrm{mM}$ each. The solutions were stirred overnight at room temperature in the dark. The 1, 5, and $10 \mathrm{~mol} \%$ UPy-BiB samples were mixed with PCLdiUPy/UPy-BiB at molar ratios of 99:1, 95:5, and 90:10, respectively, in separate vials. In a large glass Petri dish equipped with a cover, the solutions were cast $(50 \mu \mathrm{L})$ on $\varnothing 14 \mathrm{~mm}$ glass coverslips. The films were allowed to dry covered in the Petri dish for $1 \mathrm{~h}$ and subsequently air-dried overnight. Prior to characterization, the films were dried in vacuo overnight.

Preparation of Electrospun Scaffolds. PCLdiUPy and PCLdiUPy $+10 \%$ UPy-BiB were dissolved in HFIP at a total concentration of $340 \mathrm{mg} \mathrm{mL} \mathrm{m}^{-1}$. The solutions were stirred overnight at room temperature. The electrospun constructs were prepared on a climate-controlled electrospinning setup by IME Technologies (Geldrop, the Netherlands). A cylindrical mandrel rotating at 100 rpm with a diameter of $21 \mathrm{~mm}$, covered in aluminum foil was used to collect the polymer fibers. A voltage of $18 \mathrm{kV},+17 \mathrm{kV}$ on the needle and $-1 \mathrm{kV}$ on the rotating mandrel, was applied over a distance of 12 $\mathrm{cm}$ between the needle and collector. The temperature was set at 23 ${ }^{\circ} \mathrm{C}$, and relative humidity at $30 \%$. The polymer solutions were fed through a nozzle $(\varnothing 0.8 \mathrm{~mm})$ at rates of 25 and $15 \mu \mathrm{L} \mathrm{min}{ }^{-1}$ for PCLdiUPy and PCLdiUPy $+10 \%$ UPy-BiB, respectively. The aluminum foil, covered with the electrospun scaffold was removed from the mandrel and dried overnight in vacuum. Using a biopsy 
A

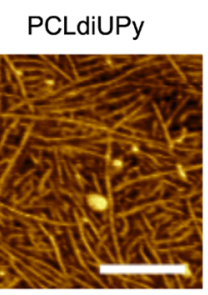
$+1 \%$ UPy-BiB

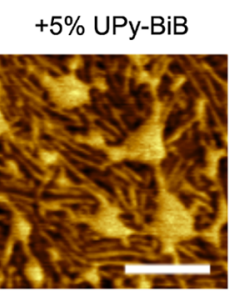
$+10 \%$ UPy-BiB

$3 \mathrm{~h}$
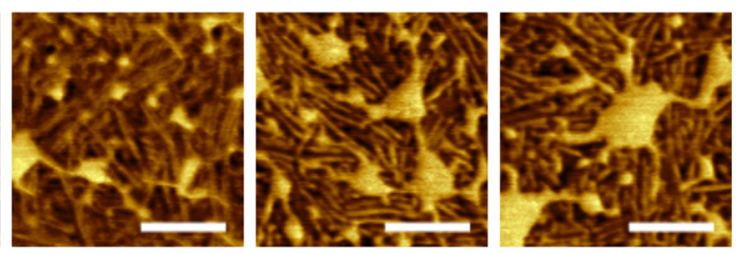

B
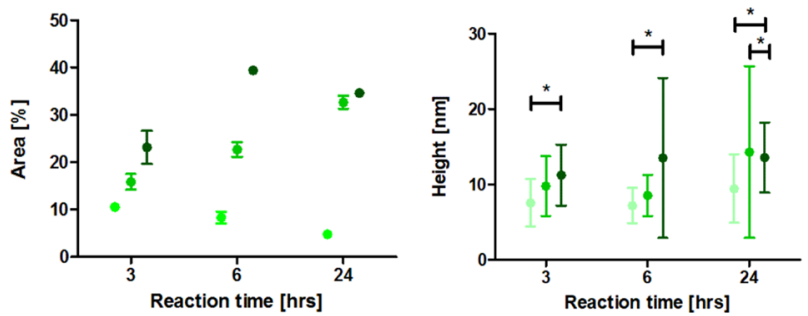

$+1 \%$ UPy-BiB

- $+5 \%$ UPy-BiB

- $+10 \%$ UPy-BiB

Figure 3. (A) Zoomed cutouts of AFM phase micrographs from the $3 \mathrm{~h}$ reaction time samples. The scale bars indicate $100 \mathrm{~nm}$. (B) Percentage of the solution-cast surface covered with SBMA domains, and the height of these domains. Data are represented as mean \pm standard deviation (SD), and relevant significant differences are indicated with an asterisk.

punch (Kai Medical), $8 \mathrm{~mm}$ circular disks were cut from the scaffold and used for polymerization and further analyses.

Surface-Initiated Atom Transfer Radical Polymerization (Figure 1C). The polymerization stock solution was prepared as follows: $\mathrm{CuCl}\left(2.60 \mathrm{mg}, 0.03 \mathrm{mmol}, 1\right.$ equiv), $\mathrm{CuCl}_{2}(31.78 \mathrm{mg}, 0.24$ mmol, 9 equiv), and bpy ( $90.24 \mathrm{mg}, 0.58 \mathrm{mmol}, 22$ equiv) were weighed in a $10 \mathrm{~mL}$ Schlenk flask equipped with a septum and a magnetic stirring bar. The flask was sealed and deoxygenated by argon flow for $30 \mathrm{~min}$. Deoxygenated Milli-Q water $(2.60 \mathrm{~mL})$ was transferred to the catalyst/ligand mixture and further deoxygenated by an argon flow until fully dissolved by stirring. The catalyst/ligand/ solvent mixture was transferred to deoxygenated sulfobetaine methacrylate ( $1.47 \mathrm{~g}, 5.25 \mathrm{mmol}, 200$ equiv) in a separate $10 \mathrm{~mL}$ Schlenk flask equipped with a septum and a magnetic stirring bar. The polymerization mixture was further deoxygenated for $15 \mathrm{~min}$ and used for the polymerization immediately.

For each polymerization condition, 10 solution-cast polymer films were immobilized in adapted 12-well Transwell inserts, from which the membrane was removed and custom-made poly(ether ether ketone) (PEEK) rings were used to mount the samples. The polymer films were degassed under a funnel with heavy argon flow for $30 \mathrm{~min}$. The polymerization was initiated by transferring the polymerization mixture $(200 \mu \mathrm{L})$ from the stock solution to each Transwell insert under exclusion of air. The polymerization was carried out at room temperature, under a moderate argon flow. The polymerization was stopped by exposing the reaction mixture to air. The polymerization mixture was removed, and each polymer film was washed with Milli- $Q$ water $(0.5 \mathrm{~mL})$ three times. Subsequently, the polymer films were removed from the inserts and thoroughly washed three times with Milli-Q water $(1 \mathrm{~mL})$ and air-dried for several hours. Prior to characterization, the films were dried in vacuo overnight. For the electrospun scaffolds, 10 samples were immobilized in similarly adapted 24-well Transwell inserts. The same polymerization conditions were applied as for solution-cast films. Post polymerization, the samples were washed by submerging the scaffolds in Milli$\mathrm{Q}$ water $(3 \mathrm{~mL})$ three times or until the strong catalyst color was imperceptible. Prior to characterization, the scaffolds were dried in vacuo overnight.

Water Contact Angle Measurements. Static water contact angles were measured using a DataPhysics OCA 30 goniometer at room temperature. Droplets of $2 \mu \mathrm{L}$ were applied on the surface, and the contact angle was measured using SCA20 software (v4.1.13) at the polymer-air-water interfaces $5 \mathrm{~s}$ after depositing the droplet, on three spots per film, for $\geq 3$ films per condition.

Scanning Electron Microscopy. Electrospun scaffolds were analyzed using an FEI Quanta 600 scanning electron microscope (SEM) and xT Microscope Control software. Images were recorded in low vacuum ( $\sim 0.6$ mbar), with water vapor of samples fixed on metal stubs with adhesive carbon conductive tape. Secondary and backscattered electrons were detected with an accelerating voltage of $10 \mathrm{kV}$ and a working distance of $10 \mathrm{~mm}$. Compound images were constructed by overlaying the resulting images from both detectors. Fiber diameters were determined from $\geq 20$ fibers from each of at least three SEM images using ImageJ software (NIH, version 1.48).

Atomic Force Microscopy. Digital Instruments Multimode and Dimension 3100 with NanoScope III controllers were used to probe the surfaces of the solution-cast films. Silicon cantilever tips with a typical tip radius of $7 \mathrm{~nm}$ were used to record phase and height images in the tapping mode regime at room temperature (PPPNCHR, 204-497 kHz, 10-130 N/m). Images were processed using Gwyddion software (version 2.43). The percentage of the surface covered by poly(sulfobetaine methacrylate) (pSBMA) domains was determined from two $1 \times 1 \mu \mathrm{m}^{2}$ phase images, the height of the domains from 40 measurements per condition, and the fiber diameter of the pristine samples from $>30$ fibers, using ImageJ software $(\mathrm{NIH}$ version 1.48).

X-ray Photoelectron Spectroscopy. X-ray photoelectron spectroscopy (XPS) measurements were performed using a Thermo Scientific K-Alpha spectrometer equipped with a monochromatic, small-spot X-ray source and a $180^{\circ}$ double-focusing, hemispherical analyzer with a 128-channel detector. Spectra were obtained using an aluminum anode $(\mathrm{Al} \mathrm{K \alpha}, 1486.7 \mathrm{eV}, 72 \mathrm{~W})$. Survey scans were measured at a pass energy of $200 \mathrm{eV}$, and region scans at a pass energy of $50 \mathrm{eV}$. Analysis and quantification of the spectra were performed using CasaXPS software on single representative measurements (version 2.3.18).

Cell Culture and Adhesion Assay. Human vena saphena cells (HVSCs), which are vascular-derived contractile and adhesive myofibroblasts, ${ }^{56}$ were harvested from the human vena saphena magna according to the Dutch guidelines for secondary use of materials. HVSCs were expanded in culture medium, which consisted of Dulbecco's modified Eagle's medium (DMEM, Gibco) supplemented with $10 \%$ fetal bovine serum (FBS), $1 \%$ glutamax (Gibco), and $1 \%$ penicillin/streptomycin and used for experiments up to passage 7 .

The solution-cast polymer films on glass were secured in adapted 12-well Transwell inserts (Corning), from which the membrane was removed and custom-made PEEK rings were used to mount the samples. Samples were sterilized under UV for $15 \mathrm{~min}$. An $8 \mathrm{~mm}$ biopsy punch (Kai Medical) was used to prepare circular samples from the electrospun constructs. These were mounted in similarly adapted 24-well Transwell inserts and sterilized under UV on both sides for $15 \mathrm{~min}$. Cells were harvested from the culture flasks using 
A

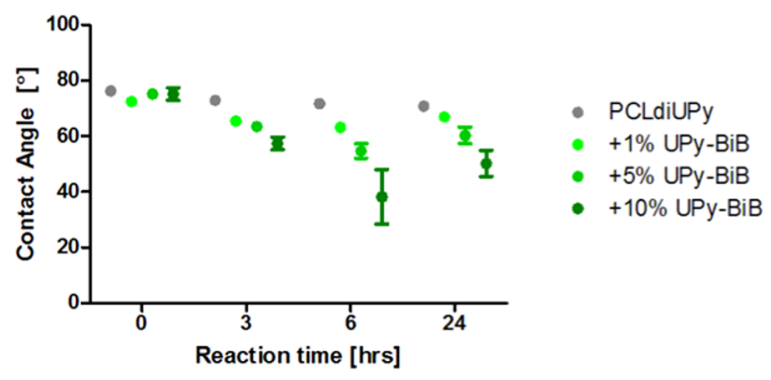

B
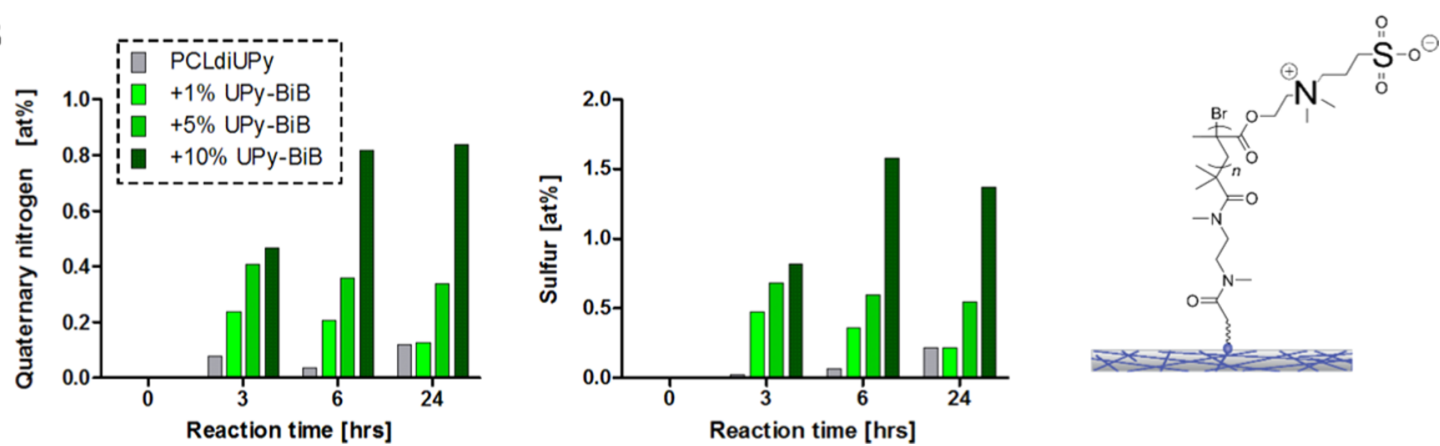

Figure 4. (A) Water contact angles measured on solution-cast films with $0,1,5$, and 10\% UPy-BiB before and after 3, 6, and $24 \mathrm{~h}$ of polymerization on the surface. Data are represented as mean \pm SD. Statistically significant differences are depicted in Figure S4B. Contribution of quaternary nitrogen and sulfur components, which are unique for the SBMA, are calculated from the XPS spectra of the solution-cast films.

trypsin/ethylenediaminetetraacetic acid (EDTA) and seeded at a concentration of 25000 cells $\mathrm{cm}^{-2}$ on the solution-cast films and 60000 cells $\mathrm{cm}^{-2}$ on the scaffold samples. After $24 \mathrm{~h}$ culture, nonadherent cells were aspirated and the samples were washed twice with phosphate-buffered saline (PBS) prior to fixation in 3.7\% formaldehyde. The actin cytoskeleton was stained with phalloidinATTO488, and nuclei were stained with 4',6-diamidino-2-phenylindole (DAPI). The samples were visualized using either a Zeiss Axiovert 200M or a Leica DMi8S. Surface coverage (i.e., the percentage of the sample surface covered by cells) was determined from a large-field-of-view image (for solution-cast films) or merged tile-scan image of the entire sample (for electrospun scaffolds) of the actin staining using ImageJ, for three samples per condition.

Statistical Analyses. Differences between groups were analyzed with nonparametric tests since it could not be determined if the groups belonged to normally distributed data due to small sample size, or normality was not proven for all groups within an experiment. Kruskal-Wallis with Dunn's post-tests, where all groups were compared, were executed using GraphPad Prism software (v5.01). $P$-values $<0.05$ were considered statistically significant.

\section{RESULTS AND DISCUSSION}

Surface Characterization. Surfaces were prepared by casting solutions of mixtures of PCLdiUPy and the UPy-BiB macroinitiator additive on glass coverslips to investigate the influence of the incorporation of the UPy-BiB additive on the surface properties. The surface morphology of the solution-cast films was investigated with AFM. In the phase images, where brighter domains indicate a harder phase, the fibrous structure resulting from the self-assembly of the UPy moieties was clearly visible (Figures 2 and S2). ${ }^{40,41,50}$ This fibrous morphology and fiber diameter (Table S1) was not altered by incorporation of up to $10 \%$ UPy-BiB additive, except for only a few small bright spots, which can be either small aggregates of UPy-BiB additive or artifacts from incomplete dissolution of polymer and/or additive. The surfaces without initiator additive were exposed to the polymerization reaction mixture for 3,6 , and $24 \mathrm{~h}$, after which the surfaces were thoroughly washed and dried before analysis. A small increase in the number of brighter domains over the exposure time was observed on these surfaces (Figures 2 and 3). These are most likely due to SBMA polymerizing in solution in the reaction mixture and adsorbing onto the surfaces, which is often observed at these specific conditions for this specific zwitterionic monomer. To verify this, an aliquot was taken from the reaction mixture, and polymer backbone peaks between 0.5 and $1 \mathrm{ppm}$ were evident in the ${ }^{1} \mathrm{H}$ NMR spectrum (Figure S3). The amount of pSBMA adsorbed onto the pristine surfaces was consistent over the entire surface (Figures 2 and S2). After $3 \mathrm{~h}$ of grafting from the supramolecular UPy$\mathrm{BiB}$ additive, brighter domains appeared increasing with UPy$\mathrm{BiB}$ concentration indicative of the presence of pSBMA and therefore successful polymerization (Figures 2 and 3). For 5 and $10 \mathrm{~mol} \%$ additive, these domains increased in size across the surface upon further reaction to $6 \mathrm{~h}$, whereas for $1 \mathrm{~mol} \%$ additive, a minimal difference was observed. When the polymerization was continued for up to $24 \mathrm{~h}$, no further increase of brighter domains was observed for all UPy-BiB concentrations compared to $6 \mathrm{~h}$. In the background, the fibrous morphology of the PCLdiUPy base material was still clearly visible in all samples. The separated domains of pSBMA were homogeneously distributed over the surfaces. Remarkably, the brighter PSBMA domains seem to originate from the hard phase in the base material, as can be observed from the zoomed-in AFM images (Figure 3). This would confirm that the initiator additive is indeed incorporated in the UPy-based assemblies and that the polymerization reaction occurred from the hard segments of this supramolecular thermoplastic elastomer.

In previous studies using SI-ATRP for functionalization with sulfobetaine polymers, a brush thickness of $\geq 20 \mathrm{~nm}$ has been reported sufficient for antifouling properties. ${ }^{17,57,58}$ From the 
A

Oh

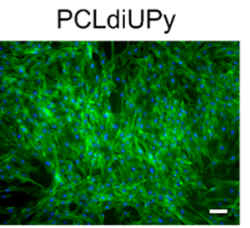

$3 h$

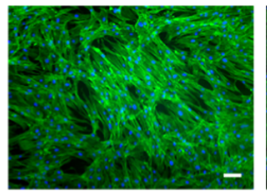

$6 h$

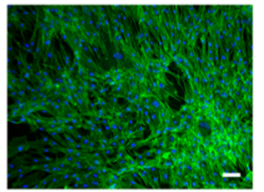

$24 \mathrm{~h}$

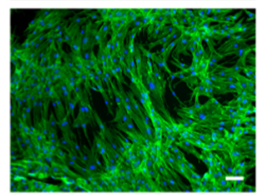

$+1 \%$ UPy-BiB
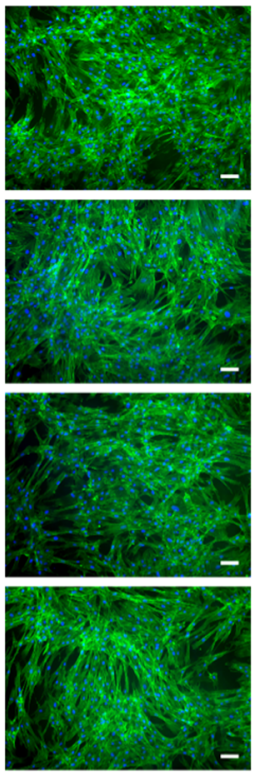
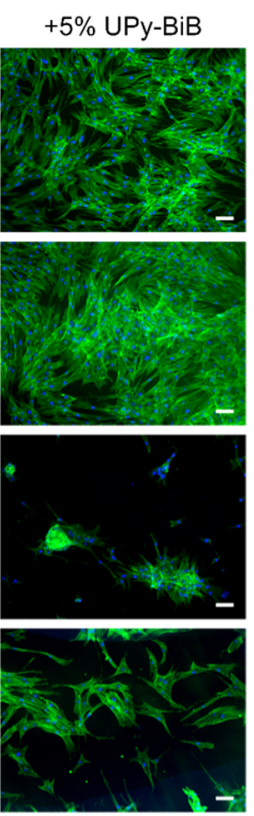
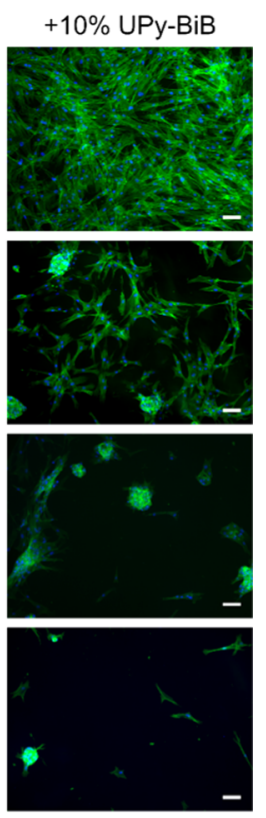

B

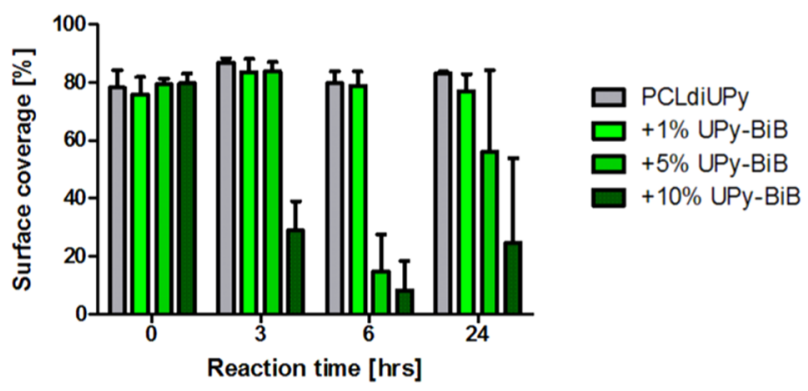

Figure 5. (A) Fluorescence micrographs of HVSCs cultured for $24 \mathrm{~h}$ on PCLdiUPy-based solution-cast films, with 0 , 1 , 5, and $10 \%$ UPy-BiB, before and after 3, 6, and $24 \mathrm{~h}$ polymerization reaction on the surface. Actin cytoskeleton is depicted in green, and nuclei in blue. The scale bars represent $100 \mu \mathrm{m}$. (B) Quantified surface coverage for the HVSCs on the solution-cast films. Data are represented as mean \pm SD. Relevant significant differences are indicated with an asterisk.

height images recorded with AFM, the height of the pSBMA domains that were observed was quantified (Figures 3 and S1). Even though the assessment of the height was relatively inaccurate due to the inherent roughness of the solution-cast surfaces (Table S2), the thickness of the pSBMA domains did not exceed $20 \mathrm{~nm}$ in general, for all UPy-BiB concentrations and polymerization times; here, an average thickness slightly over $10 \mathrm{~nm}$ was achieved for the samples.

The presence of zwitterionic SBMA on the surface of the biomaterials was evident from an emerging sulfur signal and an additional quaternary nitrogen signal in the XPS spectra (Figures 4, S5, S6, S8, and Table S3). For increasing concentrations and polymerization reaction times, the contributions of the extra nitrogen and sulfur components also increased. A surface of pure pSBMA would result in a theoretical sulfur and quaternary nitrogen concentration of approximately $5.6 \%$. The concentrations observed on the pSBMA-grafted biomaterials were significantly lower (Table S3). This could suggest that the surface coverage was indeed not complete or the thickness of polymerized pSBMA does not exceed the penetration depth of the radiation in the XPS measurements. Furthermore, the determined quaternary nitrogen component was generally lower than that for the sulfur species. Interestingly though, the reactive tertiary bromide is retained more with increasing polymerization time and thereby amount of pSBMA (Table S3 and Figure S7). A hypothetical explanation for the retention of the bromide could be that the chain-end folds back into the polymer layer, as pSBMA exhibits thermoresponsive behavior dependent on its molecular weight, $^{59}$ where it is less accessible for elimination. It is worthwhile to note that the presence of the bromide can facilitate secondary functionalization approaches, provided that the polymer chains are well solvated. ${ }^{14}$

The macroscale hydrophilicity of the surface was measured by means of WCA measurements, where a decrease in contact angle indicated the presence of hydrophilic pSBMA on the surface of the films. The addition of the UPy-BiB additive had no significant effect on the hydrophilicity of the surfaces (Figure 4). Exposure to the polymerization reaction mixture decreased the contact angle only slightly on the films without $\mathrm{UPy}-\mathrm{BiB}$, going from $76 \pm 1$ to $71 \pm 1^{\circ}$ for subsequent polymerization reaction times. For films modified with the UPy-BiB additive, the contact angle decreased both with 3 and $6 \mathrm{~h}$ polymerization reaction times and increasing additive concentration. However, contact angles of below $20^{\circ}$, reported for completely covered surfaces, ${ }^{60,61}$ were not reached, which could result from incomplete SBMA surface coverage. For the films with 1,5 , and $10 \% \mathrm{UPy}-\mathrm{BiB}$, the contact angles were again slightly higher after $24 \mathrm{~h}$ reaction time compared to $6 \mathrm{~h}$ samples. 
A
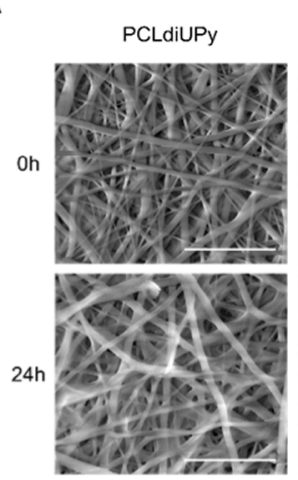

C

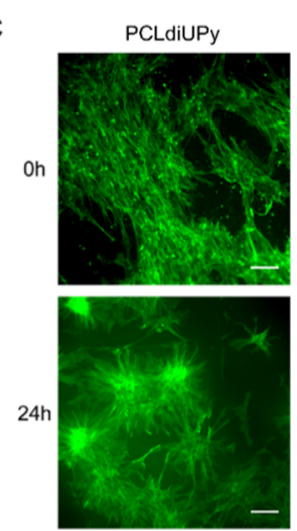

PCLdiUPy
$+10 \%$ UPy-BiB
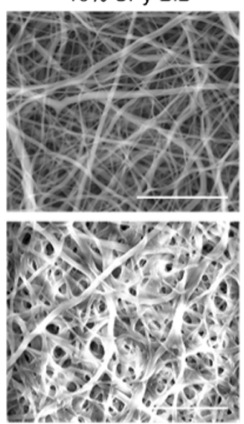

PCLdiUPy $+10 \%$ UPy-BiB

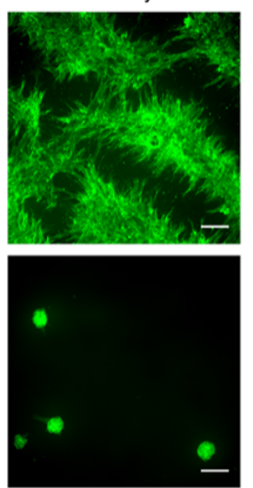

B

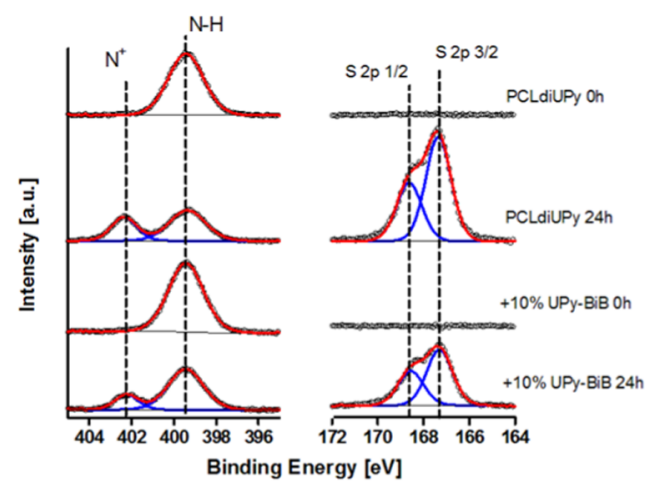

D

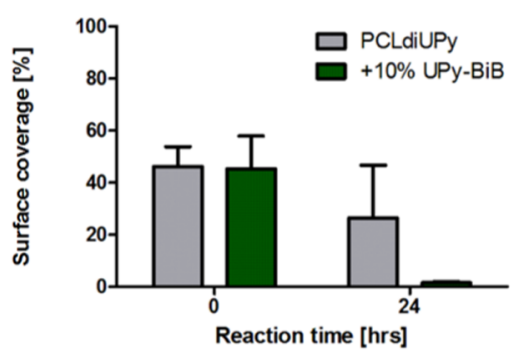

Figure 6. (A) Scanning electron micrographs of electrospun scaffolds of PCLdiUPy with 0 and 10 mol \% UPy-BiB, before and after $24 \mathrm{~h}$ reaction. The scale bars indicate $10 \mu \mathrm{m}$. (B) XPS spectra focused on nitrogen and sulfur signals. The data are represented in black, the modeled components by blue lines, and the total fit by the red line. The dashed lines indicate the location of characteristic contributions in the spectra. (C) Fluorescence micrographs of HVSCs cultured for $24 \mathrm{~h}$ on the electrospun scaffolds used for the SI-ATRP reaction. Actin cytoskeleton is depicted in green, and the scale bars indicate $100 \mu \mathrm{m}$. (D) Quantified surface coverage for the HVSCs on the electrospun scaffolds. Data are represented as mean \pm SD. Significant differences are indicated with an asterisk.

\section{Cell Adhesion on Surfaces Modified with Zwitterionic}

Polymers. To probe the functionality of the SBMA-functionalized surfaces, cell adhesion was assessed using contractile and adhesive myofibroblast-like primary human vena saphena cells (HVSCs). HVSCs were cultured on the pristine solution-cast surfaces with $0,1,5$, and $10 \% \mathrm{UPy}-\mathrm{BiB}$ and after 3,6 , and $24 \mathrm{~h}$ of polymerization of SBMA. The presence of 1,5 , and $10 \%$ UPy-BiB alone did not influence cell adhesion, as the HVSCs adhered and spread similarly to the PCLdiUPy surface without additive (Figure 5). The surfaces without the UPy-BiB additive that were exposed to the SI-ATRP reaction conditions for 3, 6, and $24 \mathrm{~h}$ showed no distinct decrease in cell adhesive properties, which indicates that neither the exposure to the polymerization reaction mixture nor the presence of aspecific adsorbed pSBMA affects cell adhesion. For the films with $1 \%$ UPy-BiB, the SBMA that was reacted to the surface had no significant effect on the adhesion and spreading of the HVSCs. After $3 \mathrm{~h}$ of the SI-ATRP reaction, the presence of pSBMA on the surface of the films with $5 \%$ UPy-BiB did not negatively influence cell adhesion. However, after $6 \mathrm{~h}$ reaction time, a striking decrease in the adhesion of the HVSCs was observed here. Patches of cells were interchanged with clusters of rounded cells on these surfaces. For the surfaces with $10 \%$ $\mathrm{UPy}-\mathrm{BiB}$, the cell adhesion decreased significantly already at 3 $\mathrm{h}$ reaction time, which was amplified after $6 \mathrm{~h}$. However, the surfaces that have been used for the polymerization reaction for $24 \mathrm{~h}$ showed an increase in the amount and variability of cell adhesion.

The observations from AFM, WCA, and XPS measurements all indicate an increased efficacy in polymerization with increasing $\mathrm{UPy}-\mathrm{BiB}$ additive concentrations up to $6 \mathrm{~h}$ of reaction time. However, for $24 \mathrm{~h}$ of reaction time, these measurements show no clear increase in polymerization efficacy compared to the $6 \mathrm{~h}$ time point. Furthermore, these results show that the pSBMA reacted from the UPy-BiB additive is functional to the extent where cell adhesion is significantly decreased, despite limited brush thickness and incomplete surface coverage as measured in dry conditions.

Incomplete surface coverage as measured with AFM could be due to aggregation of the pSBMA chains during drying. Additionally, the mismatch of the methacrylate of the SBMA monomer with our amide-based initiator is a factor. As the polymerization is initiated from the $\mathrm{UPy}-\mathrm{BiB}$, the reactivity of the growing polymer chain is increased compared to the initiator, which thereby affects uniform polymer chain growth from all of the initiating species. ${ }^{62}$ In addition to the grafting density affecting the morphology of polymer brushes, irreversible oxidation of the catalyst over time can result in incomplete polymerizations and thereby incomplete surface coverage. ${ }^{63}$ Furthermore, due to the increased length of the pSBMA chains with increasing reaction times, the UPypSBMA becomes more water-soluble and may therefore be more prone to erode from the supramolecular surface during 
the polymerization reaction and the subsequent washing steps. Additionally, pSBMA exhibits a upper critical solution temperature (UCST) behavior dependent on the molecular weight of the polymer chain, as well as the polymer concentration in aqueous solutions. ${ }^{64,65}$ Notably, in the work by O'Reilly and co-workers, a short linear pSBMA of $20 \mathrm{kDa}$ showed no thermoresponsive behavior, while long pSBMA chains of 50 and $200 \mathrm{kDa}$ only dissolved at temperatures higher than 11 and $26{ }^{\circ} \mathrm{C}$, respectively. ${ }^{59}$ As our reactions are done at room temperature, possibly long pSBMA chains aggregate and precipitate out after $6 \mathrm{~h}$ of reaction and further growth of the polymer chains is hindered.

A mechanistic insight into the surface-initiated polymerization is essential for further understanding and improved future application of the process. The detected amount of SBMA increased accordingly with the UPy-BiB additive concentration. Also, the increase in surface area occupied by pSBMA-associated domains followed this trend. However, it would be particularly interesting to evaluate the amount of actual initiation sites for polymerization, to determine grafting densities, and whether or not those correlate to the UPy-BiB concentrations and amount of detected pSBMA.

The assessment of the length of the UPy-pSBMA polymers within these domains would provide important information on the polymerization efficiency and chain length. Data on the chain length could aid in determining optimal UPy-pSBMA characteristics for robust brush formation in this material system. However, quantification of the pSBMA size was practically not feasible because of the small absolute amount of UPy-pSBMA in these small two-dimensional (2D) samples and the difficult quantification of molecular weight of zwitterionic polymer brushes on our nonideal biomaterial surfaces.

Functionality of Postmodified Electrospun Scaffolds. To further study the applicability of this postfunctionalization polymerization strategy initiated from the hard phase in a supramolecular thermoplastic elastomer, the mixture of $10 \%$ UPy-BiB additive and PCLdiUPy was electrospun into fibrous scaffolds. The scaffolds with and without $10 \%$ UPy-BiB additive had comparable morphologies and a fiber diameter of $0.36 \pm 0.15$ and $0.46 \pm 0.23 \mu \mathrm{m}$, respectively (Figure 6). The hydrophilicity increased drastically with the incorporation of $10 \mathrm{~mol} \%$ UPy-BiB additive, as the sample absorbed the water droplet within $30 \mathrm{~s}$ of droplet deposition (Figure S11). The SI-ATRP reaction was performed on the electrospun scaffolds for $24 \mathrm{~h}$ since the relative surface area of the fibrous construct is significantly higher compared to $2 \mathrm{D}$ solution-cast substrates. After polymerization, the changes in hydrophilicity of the scaffolds were not determinable due to fast wetting of the water droplet into the microporous samples. The fiber diameter of the reacted samples increased slightly to $0.43 \pm$ 0.15 and $0.58 \pm 0.24 \mu \mathrm{m}$ for the scaffolds with and without $\mathrm{UPy}-\mathrm{BiB}$, respectively. However, quantification was hampered by the presence of apparently fused fibers after the subsequent washing and drying procedures. Markedly, the scaffolds with $\mathrm{UPy}-\mathrm{BiB}$ additive that were reacted for $24 \mathrm{~h}$ charged significantly faster while measuring with scanning electron microscopy, possibly due to the presence of zwitterionic components. The XPS measurements on the scaffolds revealed the presence of quaternary nitrogen and sulfur components in the scaffolds with and without UPy-BiB that were exposed to the SI-ATRP reaction mixture, indicating that pSBMA was present in both conditions (Figures 6, S9, and Table S4).
Furthermore, an aluminum contribution was detected, attributed to the aluminum foil on which the scaffolds were prepared (Table S4 and Figure S9). The presence of physisorbed pSBMA indicates that in future experiments washing procedures can be further optimized.

The functional antifouling properties of the SBMA postmodified scaffolds were assessed with a cell adhesion assay. HVSCs were cultured for $24 \mathrm{~h}$ on the scaffolds, which adhered to and spread on the pristine scaffolds (Figure 6). The adhesion of the fibroblast-like cells was significantly decreased on the scaffolds with UPy-BiB that were reacted for $24 \mathrm{~h}$, whereas the decrease in cell adhesion was less defined for the scaffolds without the UPy-BiB additive. This shows that, even though the XPS measurements identified the presence of pSBMA on the scaffolds without UPy-BiB, as well as on the scaffolds with UPy-BiB, the functional antifouling properties of the polymerized UPy-pSBMA are clearly superior.

General Discussion. One interesting observation from both solution-cast films and electrospun scaffolds was the apparent improved stability of the tertiary bromide on the polymerized pSBMA molecules, compared to the UPy-BiB initiator. In previous reports, the bromide signal is not present after polymerization, where reported tertiary bromide concentrations prior to reacting range from 0.81 to $1.9 .^{18,19,66}$ Due to the noncovalent nature of the incorporation of the UPypSBMA after polymerization, the stability of the postfunctionalized substrates should be subject of future studies. The polymerization reaction time of $24 \mathrm{~h}$ is particularly long, compared to the literature. ${ }^{17}$ In the current setup, a color change in the polymerization reaction mixture indicated irreversible oxidation of the copper catalyst between the 6 and $24 \mathrm{~h}$ time points. Therefore, future efforts will focus on the optimization of the setup to facilitate a continuous inert atmosphere for SI-ATRP, as well as switching to a more oxygen-tolerant polymerization to have better control over the polymer molecular weights, dispersities, and polymer chainend livingness.

\section{CONCLUSIONS AND OUTLOOK}

For the first time, the successful ATRP of zwitterionic SBMA monomers from a macroinitiator additive incorporated in the hard phase of a supramolecular elastomeric material is reported here. Surface analysis by AFM, XPS, and WCA measurements, as well as cell adhesion assays, show an initiator concentration and reaction-time-dependent polymerization efficacy. Cell adhesion was decreased significantly for surfaces with 5 and $10 \mathrm{~mol} \% \mathrm{UPy}-\mathrm{BiB}$ additive and $6 \mathrm{~h}$ reaction time. The noncell adhesive properties could be translated to a functional electrospun scaffold with $10 \mathrm{~mol} \% \mathrm{UPy}-\mathrm{BiB}$. These results serve as the first step toward the fabrication of scaffolds or membranes that can be functionally postmodified via polymerization through the simple inclusion of a macroinitiator additive in the hard phase of the structural base material.

The electrospun scaffolds postmodified with zwitterionic polymers offer many possibilities, mostly due to the properties of the zwitterionic compounds. The responsivity to varying salt concentrations could be employed to tune the porosity of the scaffolds reversibly. ${ }^{29}$ Additionally, the interesting UCST behavior of pSBMA can give similar switchable properties. ${ }^{67}$

Moreover, a popular approach featuring antifouling materials involves further modification with specific bioactive moieties. ${ }^{68}$ This could be achieved through the reaction of bioactive moieties to reactive monomers that were co-polymerized 
during the modification with the antifouling layer, ${ }^{66}$ or by endfunctionalizing the antifouling brush, making use of the reactive nature of the halide-capped polymer ends. ${ }^{69,70}$

Additionally, with biomedical applications in mind, the amount of copper used in the procedure described here could be decreased significantly through application of greener radical polymerization strategies, such as activator regenerated ATRP. ${ }^{1-78}$

\section{ASSOCIATED CONTENT}

\section{SI Supporting Information}

The Supporting Information is available free of charge at https://pubs.acs.org/doi/10.1021/acs.macromol.0c00160.

Reaction schemes, additional AFM results, NMR data, and additional XPS spectra and data (PDF)

\section{AUTHOR INFORMATION}

\section{Corresponding Author}

Patricia Y. W. Dankers - Institute for Complex Molecular Systems, Laboratory for Cell and Tissue Engineering, Department of Biomedical Engineering, and Laboratory of Chemical Biology, Department of Biomedical Engineering, Eindhoven University of Technology, 5600 MB Eindhoven, The Netherlands; 10 orcid.org/0000-0002-8997-181X; Email: p.y.w.dankers@tue.nl

\section{Authors}

Bastiaan D. Ippel - Institute for Complex Molecular Systems and Laboratory for Cell and Tissue Engineering, Department of Biomedical Engineering, Eindhoven University of Technology, 5600 MB Eindhoven, The Netherlands

Muhabbat I. Komil - Institute for Complex Molecular Systems and Laboratory of Chemical Biology, Department of Biomedical Engineering, Eindhoven University of Technology, 5600 MB Eindhoven, The Netherlands

Paul A. A. Bartels - Institute for Complex Molecular Systems and Laboratory of Chemical Biology, Department of Biomedical Engineering, Eindhoven University of Technology, 5600 MB Eindhoven, The Netherlands

Serge H. M. Söntjens - SyMO-Chem BV, 5612 AZ Eindhoven, The Netherlands

Roy J. E. A. Boonen - Institute for Complex Molecular Systems and Laboratory of Chemical Biology, Department of Biomedical Engineering, Eindhoven University of Technology, 5600 MB Eindhoven, The Netherlands

Maarten M. J. Smulders - Laboratory of Organic Chemistry, Wageningen University, 6708 WE Wageningen, The Netherlands; (1) orcid.org/0000-0002-6855-0426

Complete contact information is available at:

https://pubs.acs.org/10.1021/acs.macromol.0c00160

\section{Author Contributions}

\#B.D.I. and M.I.K. contributed equally to this work.

\section{Notes}

The authors declare no competing financial interest.

\section{ACKNOWLEDGMENTS}

The authors would like to acknowledge H. M. Janssen for useful discussions, M. G. H. Verhoeven for collaboration on the XPS measurements, and P. P. K. H. Fransen for the synthesis of the UPy-COOH synthon. The ICMS Animation Studio is acknowledged for the design of some of the cartoons.
This work was funded by the Ministry of Education, Culture and Science (Gravity Program 024.001.03) and ZonMW as part of the LSH 2Treat program (Project no. 436001003).

\section{REFERENCES}

(1) Banerjee, I.; Pangule, R. C.; Kane, R. S. Antifouling Coatings: Recent Developments in the Design of Surfaces That Prevent Fouling by Proteins, Bacteria, and Marine Organisms. Adv. Mater. 2011, 23, $690-718$

(2) Yu, Q.; Zhang, Y.; Wang, H.; Brash, J.; Chen, H. Anti-Fouling Bioactive Surfaces. Acta Biomater. 2011, 7, 1550-1557.

(3) Wei, Q.; Becherer, T.; Angioletti-Uberti, S.; Dzubiella, J.; Wischke, C.; Neffe, A. T.; Lendlein, A.; Ballauff, M.; Haag, R. Protein Interactions with Polymer Coatings and Biomaterials. Angew. Chem., Int. Ed. 2014, 53, 8004-8031.

(4) Ippel, B. D.; Dankers, P. Y. W. Introduction of Nature's Complexity in Engineered Blood-Compatible Biomaterials. Adv. Healthcare Mater. 2018, 7, No. 1700505.

(5) Chen, S.; Li, L.; Zhao, C.; Zheng, J. Surface Hydration: Principles and Applications toward Low-Fouling/Nonfouling Biomaterials. Polymer 2010, 51, 5283-5293.

(6) Schlenoff, J. B. Zwitteration: Coating Surfaces with Zwitterionic Functionality to Reduce Nonspecific Adsorption. Langmuir 2014, 30, 9625-9636.

(7) Jiang, S.; Cao, Z. Ultralow-Fouling, Functionalizable, and Hydrolyzable Zwitterionic Materials and Their Derivatives for Biological Applications. Adv. Mater. 2010, 22, 920-932.

(8) Chen, W.-L.; Cordero, R.; Tran, H.; Ober, C. K. 50th Anniversary Perspective: Polymer Brushes: Novel Surfaces for Future Materials. Macromolecules 2017, 50, 4089-4113.

(9) Shao, Q.; Jiang, S. Molecular Understanding and Design of Zwitterionic Materials. Adv. Mater. 2015, 27, 15-26.

(10) Edmondson, S.; Osborne, V. L.; Huck, W. T. S. Polymer Brushes via Surface-Initiated Polymerizations. Chem. Soc. Rev. 2004 33, 14.

(11) Yuan, L.; Yu, Q.; Li, D.; Chen, H. Surface Modification to Control Protein/Surface Interactions. Macromol. Biosci. 2011, 11, 1031-1040.

(12) Krishnamoorthy, M.; Hakobyan, S.; Ramstedt, M.; Gautrot, J. E. Surface-Initiated Polymer Brushes in the Biomedical Field: Applications in Membrane Science, Biosensing, Cell Culture, Regenerative Medicine and Antibacterial Coatings. Chem. Rev. 2014, 114, 10976-11026.

(13) Barbey, R.; Lavanant, L.; Paripovic, D.; Schüwer, N.; Sugnaux, C.; Tugulu, S.; Klok, H. Polymer Brushes via Surface-Initiated Controlled Radical Polymerization: Synthesis, Characterization, Properties, and Applications. Chem. Rev. 2009, 109, 5437-5527.

(14) Zoppe, J. O.; Ataman, N. C.; Mocny, P.; Wang, J.; Moraes, J.; Klok, H.-A. Surface-Initiated Controlled Radical Polymerization: State-of-the-Art, Opportunities, and Challenges in Surface and Interface Engineering with Polymer Brushes. Chem. Rev. 2017, 117, $1105-1318$

(15) Wei, Y.; Zhang, J.; Feng, X.; Liu, D. Bioactive Zwitterionic Polymer Brushes Grafted from Silicon Wafers via SI-ATRP for Enhancement of Antifouling Properties and Endothelial Cell Selectivity. J. Biomater. Sci., Polym. Ed. 2017, 28, 2101-2116.

(16) Zhang, Z.; Chen, S.; Jiang, S. Dual-Functional Biomimetic Materials: Nonfouling Poly(Carboxybetaine) with Active Functional Groups for Protein Immobilization. Biomacromolecules 2006, 7, 3311-3315.

(17) van Andel, E.; Lange, S. C.; Pujari, S. P.; Tijhaar, E. J.; Smulders, M. M. J.; Savelkoul, H. F. J.; Zuilhof, H. Systematic Comparison of Zwitterionic and Non-Zwitterionic Antifouling Polymer Brushes on a Bead-Based Platform. Langmuir 2019, 35, $1181-1191$

(18) Kuang, J.; Messersmith, P. B. Universal Surface-Initiated Polymerization of Antifouling Zwitterionic Brushes Using a MusselMimetic Peptide Initiator. Langmuir 2012, 28, 7258-7266. 
(19) Fan, X.; Lin, L.; Dalsin, J. L.; Messersmith, P. B. Biomimetic Anchor for Surface-Initiated Polymerization from Metal Substrates. J. Am. Chem. Soc. 2005, 127, 15843-15847.

(20) Surman, F.; Riedel, T.; Bruns, M.; Kostina, N. Y.; Sedláková, Z.; Rodriguez-Emmenegger, C. Polymer Brushes Interfacing Blood as a Route toward High Performance Blood Contacting Devices. Macromol. Biosci. 2015, 15, 636-646.

(21) Kostina, N. Y.; Pop-Georgievski, O.; Bachmann, M.; Neykova, N.; Bruns, M.; Michálek, J.; Bastmeyer, M.; Rodriguez-Emmenegger, C. Non-Fouling Biodegradable Poly $(\epsilon$-Caprolactone $)$ Nanofibers for Tissue Engineering. Macromol. Biosci. 2016, 16, 83-94.

(22) Gualandi, C.; Vo, C. D.; Focarete, M. L.; Scandola, M.; Pollicino, A.; Di Silvestro, G.; Tirelli, N. Advantages of SurfaceInitiated ATRP (SI-ATRP) for the Functionalization of Electrospun Materials. Macromol. Rapid Commun. 2013, 34, 51-56.

(23) Yue, W. W.; Li, H. J.; Xiang, T.; Qin, H.; Sun, S. D.; Zhao, C. S. Grafting of Zwitterion from Polysulfone Membrane via SurfaceInitiated ATRP with Enhanced Antifouling Property and Biocompatibility. J. Membr. Sci. 2013, 446, 79-91.

(24) Liu, P.; Huang, T.; Liu, P.; Shi, S.; Chen, Q.; Li, L.; Shen, J. Zwitterionic Modification of Polyurethane Membranes for Enhancing the Anti-Fouling Property. J. Colloid Interface Sci. 2016, 480, 91-101. (25) Yuan, H.; Qian, B.; Zhang, W.; Lan, M. Protein Adsorption Resistance of PVP-Modified Polyurethane Film Prepared by SurfaceInitiated Atom Transfer Radical Polymerization. Appl. Surf. Sci. 2016, 363, 483-489.

(26) Sun, Q.; Su, Y.; Ma, X.; Wang, Y.; Jiang, Z. Improved Antifouling Property of Zwitterionic Ultrafiltration Membrane Composed of Acrylonitrile and Sulfobetaine Copolymer. J. Membr. Sci. 2006, 285, 299-305.

(27) Wang, L.; Su, Y.; Zheng, L.; Chen, W.; Jiang, Z. Highly Efficient Antifouling Ultrafiltration Membranes Incorporating Zwitterionic Poly([3-(Methacryloylamino)Propyl]-Dimethyl(3-Sulfopropyl) Ammonium Hydroxide). J. Membr. Sci. 2009, 340, 164-170.

(28) Li, J.-H.; Li, M.-Z.; Miao, J.; Wang, J.-B.; Shao, X.-S.; Zhang, Q.-Q. Improved Surface Property of PVDF Membrane with Amphiphilic Zwitterionic Copolymer as Membrane Additive. Appl. Surf. Sci. 2012, 258, 6398-6405.

(29) Zhao, Y.-F.; Zhang, P.-B.; Sun, J.; Liu, C.-J.; Zhu, L.-P.; Xu, Y.Y. Electrolyte-Responsive Polyethersulfone Membranes with Zwitterionic Polyethersulfone-Based Copolymers as Additive. J. Membr. Sci. 2016, 510, 306-313.

(30) Fang, L.-F.; Jeon, S.; Kakihana, Y.; Kakehi, J.; Zhu, B.-K.; Matsuyama, H.; Zhao, S. Improved Antifouling Properties of Polyvinyl Chloride Blend Membranes by Novel Phosphate BasedZwitterionic Polymer Additive. J. Membr. Sci. 2017, 528, 326-335.

(31) Kaner, P.; Rubakh, E.; Kim, D. H.; Asatekin, A. ZwitterionContaining Polymer Additives for Fouling Resistant Ultrafiltration Membranes. J. Membr. Sci. 2017, 533, 141-159.

(32) Rodda, A. E.; Ercole, F.; Glattauer, V.; Nisbet, D. R.; Healy, K. E.; Dove, A. P.; Meagher, L.; Forsythe, J. S. Controlling IntegrinBased Adhesion to a Degradable Electrospun Fibre Scaffold via SIATRP. J. Mater. Chem. B 2016, 4, 7314-7322.

(33) Duque-Sánchez, L.; Brack, N.; Postma, A.; Pigram, P. J.; Meagher, L. Optimisation of Grafting of Low Fouling Polymers from Three-Dimensional Scaffolds: Via Surface-Initiated $\mathrm{Cu}(0)$ Mediated Polymerisation. J. Mater. Chem. B 2018, 6, 5896-5909.

(34) Harrison, R. H.; Steele, J. A. M.; Chapman, R.; Gormley, A. J.; Chow, L. W.; Mahat, M. M.; Podhorska, L.; Palgrave, R. G.; Payne, D. J.; Hettiaratchy, S. P.; Dunlop, I. E.; Stevens, M. M. Modular and Versatile Spatial Functionalization of Tissue Engineering Scaffolds through Fiber-Initiated Controlled Radical Polymerization. Adv. Funct. Mater. 2015, 25, 5748-5757.

(35) Duque Sánchez, L.; Brack, N.; Postma, A.; Pigram, P. J.; Meagher, L. Surface Modification of Electrospun Fibres for Biomedical Applications: A Focus on Radical Polymerization Methods. Biomaterials 2016, 106, 24-45.

(36) Chin, S. M.; Synatschke, C. V.; Liu, S.; Nap, R. J.; Sather, N. A.; Wang, Q.; Álvarez, Z.; Edelbrock, A. N.; Fyrner, T.; Palmer, L. C.;
Szleifer, I.; Olvera De La Cruz, M.; Stupp, S. I. CovalentSupramolecular Hybrid Polymers as Muscle-Inspired Anisotropic Actuators. Nat. Commun. 2018, 9, No. 2395.

(37) Sijbesma, R. P.; Beijer, F. H.; Brunsveld, L.; Folmer, B. J.; Hirschberg, J. H.; Lange, R. F.; Lowe, J. K.; Meijer, E. W. Reversible Polymers Formed from Self-Complementary Monomers Using Quadruple Hydrogen Bonding. Science 1997, 278, 1601-1604.

(38) Beijer, F. H.; Sijbesma, R. P.; Kooijman, H.; Spek, A. L.; Meijer, E. W. Strong Dimerization of Ureidopyrimidones via Quadruple Hydrogen Bonding. J. Am. Chem. Soc. 1998, 120, 6761-6769.

(39) Folmer, B. J. B.; Sijbesma, R. P.; Versteegen, R. M.; Van Der Rijt, J. A. J.; Meijer, E. W. Supramolecular Polymer Materials: Chain Extension of Telechelic Polymers Using a Reactive HydrogenBonding Synthon. Adv. Mater. 2000, 12, 874-878.

(40) Appel, W. P. J.; Portale, G.; Wisse, E.; Dankers, P. Y. W.; Meijer, E. W. Aggregation of Ureido-Pyrimidinone Supramolecular Thermoplastic Elastomers into Nanofibers: A Kinetic Analysis. Macromolecules 2011, 44, 6776-6784.

(41) Kautz, H.; van Beek, D. J. M.; Sijbesma, R. P.; Meijer, E. W. Cooperative End-to-End and Lateral Hydrogen-Bonding Motifs in Supramolecular Thermoplastic Elastomers. Macromolecules 2006, 39, 4265-4267.

(42) Pape, A. C. H.; Ippel, B. D.; Dankers, P. Y. W. Cell and Protein Fouling Properties of Polymeric Mixtures Containing Supramolecular Poly(Ethylene Glycol) Additives. Langmuir 2017, 33, 4076-4082.

(43) Van Almen, G. C.; Talacua, H.; Ippel, B. D.; Mollet, B. B. B.; Ramaekers, M.; Simonet, M.; Smits, A. I. P. M.; Bouten, C. V. C.; Kluin, J.; Dankers, P. Y. W. Development of Non-Cell Adhesive Vascular Grafts Using Supramolecular Building Blocks. Macromol. Biosci. 2016, 16, 350-362.

(44) Mollet, B. B.; Comellas-Aragonès, M.; Spiering, A. J. H.; Söntjens, S. H. M.; Meijer, E. W.; Dankers, P. Y. W. A Modular Approach to Easily Processable Supramolecular Bilayered Scaffolds with Tailorable Properties. J. Mater. Chem. B 2014, 2, 2483-2493.

(45) Ippel, B. D.; Keizer, H. M.; Dankers, P. Y. W. Supramolecular Antifouling Additives for Robust and Efficient Functionalization of Elastomeric Materials: Molecular Design Matters. Adv. Funct. Mater. 2019, 29, No. 1805375 .

(46) Zaccaria, S.; van Gaal, R. C.; Riool, M.; Zaat, S. A. J.; Dankers, P. Y. W. Antimicrobial Peptide Modification of Biomaterials Using Supramolecular Additives. J. Polym. Sci., Part A: Polym. Chem. 2018, 56, 1926-1934.

(47) Dankers, P. Y. W.; Harmsen, M. C.; Brouwer, L. A.; van Luyn, M. J. A.; Meijer, E. W. A Modular and Supramolecular Approach to Bioactive Scaffolds for Tissue Engineering. Nat. Mater. 2005, 4, 568574

(48) Spaans, S.; Fransen, P. P. K. H.; Ippel, B. D.; de Bont, D. F. A.; Keizer, H. M.; Bax, N. A. M. A. M.; Bouten, C. V. C.; Dankers, P. Y. W. Supramolecular Surface Functionalization via Catechols for the Improvement of Cell-Material Interactions. Biomater. Sci. 2017, 5, $1541-1548$

(49) Muylaert, D. E. P.; van Almen, G. C.; Talacua, H.; Fledderus, J. O.; Kluin, J.; Hendrikse, S. I. S.; van Dongen, J. L. J.; Sijbesma, E.; Bosman, A. W.; Mes, T.; Thakkar, S. H.; Smits, A. I. P. M.; Bouten, C. V. C.; Dankers, P. Y. W.; Verhaar, M. C. Early In-Situ Cellularization of a Supramolecular Vascular Graft Is Modified by Synthetic Stromal Cell-Derived Factor-1 $\alpha$ Derived Peptides. Biomaterials 2016, 76, 187-195.

(50) Appel, W. P. J.; Meijer, E. W.; Dankers, P. Y. W. Enzymatic Activity at the Surface of Biomaterials via Supramolecular Anchoring of Peptides: The Effect of Material Processing. Macromol. Biosci. 2011, 11, 1706-1712.

(51) Goor, O. J. G. M.; Brouns, J. E. P.; Dankers, P. Y. W. Introduction of Anti-Fouling Coatings at the Surface of Supramolecular Elastomeric Materials via Post-Modification of Reactive Supramolecular Additives. Polym. Chem. 2017, 8, 5228-5238.

(52) Goor, O. J. G. M.; Keizer, H. M.; Bruinen, A. L.; Schmitz, M. G. J.; Versteegen, R. M.; Janssen, H. M.; Heeren, R. M. A.; Dankers, P. Y. 
W. Efficient Functionalization of Additives at Supramolecular Material Surfaces. Adv. Mater. 2017, 29, No. 1604652.

(53) Wisse, E.; Spiering, A. J. H.; Dankers, P. Y. W.; Mezari, B.; Magusin, P. C. M. M.; Meijer, E. W. Multicomponent Supramolecular Thermoplastic Elastomer with Peptide-Modified Nanofibers. J. Polym. Sci., Part A: Polym. Chem. 2011, 49, 1764-1771.

(54) De Feijter, I.; Goor, O. J. G. M.; Hendrikse, S. I. S.; ComellasAragonès, M.; Söntjens, S. H. M.; Zaccaria, S.; Fransen, P. P. K. H.; Peeters, J. W.; Milroy, L. G.; Dankers, P. Y. W. Solid-Phase-Based Synthesis of Ureidopyrimidinone-Peptide Conjugates for Supramolecular Biomaterials. Synlett 2015, 26, 2707-2713.

(55) Keller, R. N.; Wrcoff, H. D.; Marchi, L. E. Copper(I) Chloride. Inorganic Syntheses; McGraw-Hill Book Company, Inc., 1946; Vol. 2, pp $1-4$.

(56) Mol, A.; Rutten, M. C. M.; Driessen, N. J. B.; Bouten, C. V. C.; Zund, G.; Baaijens, F. P. T.; Hoerstrup, S. P. Autologous Human Tissue-Engineered Heart Valves: Prospects for Systemic Application. Circulation 2006, 114, I152-I158.

(57) Nguyen, A. T.; Baggerman, J.; Paulusse, J. M. J.; van Rijn, C. J. M.; Zuilhof, H. Stable Protein-Repellent Zwitterionic Polymer Brushes Grafted from Silicon Nitride. Langmuir 2011, 27, 25872594.

(58) Blaszykowski, C.; Sheikh, S.; Thompson, M. A Survey of Stateof-the-Art Surface Chemistries to Minimize Fouling from Human and Animal Biofluids. Biomater. Sci. 2015, 3, 1335-1370.

(59) Willcock, H.; Lu, A.; Hansell, C. F.; Chapman, E.; Collins, I. R.; O'Reilly, R. K. One-Pot Synthesis of Responsive Sulfobetaine Nanoparticles by RAFT Polymerisation: The Effect of Branching on the UCST Cloud Point. Polym. Chem. 2014, 5, 1023-1030.

(60) Cheng, N.; Brown, A. A.; Azzaroni, O.; Huck, W. T. S. Thickness-Dependent Properties of Polyzwitterionic Brushes. Macromolecules 2008, 41, 6317-6321.

(61) Emmenegger, C. R.; Brynda, E.; Riedel, T.; Sedlakova, Z.; Houska, M.; Alles, A. B. Interaction of Blood Plasma with Antifouling Surfaces. Langmuir 2009, 25, 6328-6333.

(62) Tang, W.; Matyjaszewski, K. Effects of Initiator Structure on Activation Rate Constants in ATRP. Macromolecules 2007, 40, 18581863.

(63) Tsujii, Y.; Ohno, K.; Yamamoto, S.; Goto, A.; Fukuda, T. Structure and Properties of High-Density Polymer Brushes Prepared by Surface-Initiated Living Radical Polymerization. In Surface-Initiated Polymerization; Jordan, R., Ed.; Springer: Berlin, Heidelberg, 2006; pp $1-45$.

(64) Niskanen, J.; Tenhu, H. How to Manipulate the Upper Critical Solution Temperature (UCST)? Polym. Chem. 2017, 8, 220-232.

(65) Polzer, F.; Heigl, J.; Schneider, C.; Ballauff, M.; Borisov, O. V. Synthesis and Analysis of Zwitterionic Spherical Polyelectrolyte Brushes in Aqueous Solution. Macromolecules 2011, 44, 1654-1660.

(66) Lange, S. C.; van Andel, E.; Smulders, M. M. J.; Zuilhof, H. Efficient and Tunable Three-Dimensional Functionalization of Fully Zwitterionic Antifouling Surface Coatings. Langmuir 2016, 32, 10199-10205.

(67) Azzaroni, O.; Brown, A. A.; Huck, W. T. S. UCST Wetting Transitions of Polyzwitterionic Brushes Driven by Self-Association. Angew. Chem., Int. Ed. 2006, 45, 1770-1774.

(68) Baggerman, J.; Smulders, M. M. J.; Zuilhof, H. Romantic Surfaces: A Systematic Overview of Stable, Biospecific, and Antifouling Zwitterionic Surfaces. Langmuir 2019, 35, 1072-1084.

(69) Huang, C. J.; Brault, N. D.; Li, Y.; Yu, Q.; Jiang, S. Controlled Hierarchical Architecture in Surface-Initiated Zwitterionic Polymer Brushes with Structurally Regulated Functionalities. Adv. Mater. 2012, 24, 1834-1837.

(70) Nguyen, A. T.; Baggerman, J.; Paulusse, J. M. J.; Zuilhof, H.; van Rijn, C. J. M. Bioconjugation of Protein-Repellent Zwitterionic Polymer Brushes Grafted from Silicon Nitride. Langmuir 2012, 28, 604-610.

(71) Matyjaszewski, K.; Hongchen, D.; Jakubowski, W.; Pietrasik, J.; Kusumo, A. Grafting from Surfaces for "Everyone": ARGET ATRP in the Presence of Air. Langmuir 2007, 23, 4528-4531.
(72) Hong, D.; Hung, H. C.; Wu, K.; Lin, X.; Sun, F.; Zhang, P.; Liu, S.; Cook, K. E.; Jiang, S. Achieving Ultralow Fouling under Ambient Conditions via Surface-Initiated ARGET ATRP of Carboxybetaine. ACS Appl. Mater. Interfaces 2017, 9, 9255-9259.

(73) Wang, M.; Yuan, J.; Huang, X.; Cai, X.; Li, L.; Shen, J. Grafting of Carboxybetaine Brush onto Cellulose Membranes via SurfaceInitiated ARGET-ATRP for Improving Blood Compatibility. Colloids Surf., B 2013, 103, 52-58.

(74) Jakubowski, W.; Matyjaszewski, K. Activators Regenerated by Electron Transfer for Atom-Transfer Radical Polymerization of (Meth)Acrylates and Related Block Copolymers. Angew. Chem., Int. Ed. 2006, 45, 4482-4486.

(75) Min, K.; Gao, H.; Matyjaszewski, K. Use of Ascorbic Acid as Reducing Agent for Synthesis of Well-Defined Polymers by ARGET ATRP. Macromolecules 2007, 40, 1789-1791.

(76) Konkolewicz, D.; Magenau, A. J. D.; Averick, S. E.; Simakova, A.; He, H.; Matyjaszewski, K. ICAR ATRP with Ppm Cu Catalyst in Water. Macromolecules 2012, 45, 4461-4468.

(77) Konkolewicz, D.; Krys, P.; Góis, J. R.; Mendonça, P. V.; Zhong, M.; Wang, Y.; Gennaro, A.; Isse, A. A.; Fantin, M.; Matyjaszewski, K. Aqueous RDRP in the Presence of Cu0: The Exceptional Activity of CuI Confirms the SARA ATRP Mechanism. Macromolecules 2014, 47, 560-570.

(78) Pan, X.; Malhotra, N.; Simakova, A.; Wang, Z.; Konkolewicz, D.; Matyjaszewski, K. Photoinduced Atom Transfer Radical Polymerization with Ppm-Level $\mathrm{Cu}$ Catalyst by Visible Light in Aqueous Media. J. Am. Chem. Soc. 2015, 137, 15430-15433. 